\title{
Grouping by proximity: Selective attention measures
}

\author{
JAMES R. POMERANTZ and STEVEN D. SCHWAITZBERG \\ The Johns Hopkins University, Baltimore, Maryland 21218
}

\begin{abstract}
The role of element proximity in perceptual grouping was examined in tasks requiring speeded discrimination of two-element visual patterns. Grouping of two elements was defined as the failure of attention to be focused on one element selectively in filtering tasks where only that one element was relevant to the discrimination. Failure of selective attention was measured by the degree of interference caused by variation of the irrelevant element. Grouping was shown to diminish monotonically as the spacing between two elements was increased. At a given spacing, grouping could be reduced or eliminated by the introduction of a third element into the stimulus field, presumably because the addition of this element triggered a reorganization of the perceptual field into a new grouping structure. Grouping appeared to facilitate performance on condensation tasks requiring distributed attention, to the degree that the condensation tasks were actually easier than the filtering tasks at close proximities. Paradoxically, for some tasks, moving an irrelevant element away from a relevant one actually impaired performance, suggesting that paying attention to irrelevant information could be beneficial. This result, if generalizable, suggests that grouping be conceptualized not as an automatic process under preattentive control but as an optional process under strategic control.
\end{abstract}

This paper is concerned with two aspects of the problem of perceptual organization of visual arrays (Wertheimer, 1923). The first is the degree to which perceptual organization is under the control of specific parameters of the stimulus array, as opposed to cognitive (strategic) factors within the observer. The second is the establishment of experimental measures of organizational processes. This latter issue is much more than simply a methodological one, for in order to measure organization a theory is required that explains just what organization means, and how and where organization enters into the processing of perceptual information.

The Gestalt "laws" of course represented an attempt to answer the first question. Certain stimulus variables, some well defined (proximity) and some not so well defined (similarity), can be shown to have clear effects on the way an observer reports his perception of stimulus arrays. The conclusions one can draw from these demonstrations, as compelling as the perceptual effects may be, are linited, however, to the domain of phenomenology and say little about the underlying processes that shape our perceptual experiences. What is required are performance measures, rather than subjective reports. of perceptual organization.

Pomerantz and Garner (1973) argued that if organization is more than an epiphenomenon, there

This research was supported in part' by Grant MH 14229 from the National Institute of Mental Health to Yale University and in part by Biomedical Sctences Support Grant 5S05 RR 07041-09 awarded to The Johns Hopkins University by the Division of Research Resources, DHEW. The authors thank W. R. Garner for his suggestions. Requests for reprints should be sent to James Pomerantz, Department of Psychology. The Johns Hopkins University, Baltimore, Maryland 21218. should be consequences of such organization in the performance of visual information processing tasks. More specifically, we reasoned that any organizational process should affect the distribution of attention across the elements in a stimulus array. If two elements are grouped together in the perceptual process to form a single unit, then selective attention to one of the elements in that unit should be difficult, or perhaps impossible, without breaking up the unit through some sort of perceptual reorganization. If two elements are not grouped into a single unit, then selective attention to either element should be easy. This assumption made it possible to look for organizational effects with processing tasks used in selective attention research (Egeth, 1967; Garner \& Felfoldy, 1970). Here the subject is presented with several informational sources simultaneously (e.g., several stimuli or a single stimulus which varies in several attributes). His task is to respond to the one relevant source and ignore (or filter) the other, irrelevant, sources. The experimental question is often the degree to which variation in the irrelevant sources interferes with processing of the relevant source. If no interference is observed, selective attention has succeeded; otherwise, it has failed (Garner, 1974).

Pomerantz and Garner (1973) used the stimuli in Figure 1a to test this hypothesis, that perceptual grouping implies a failure of selective attention. In one experiment, the subject was presented with a sequence of stimuli drawn from the set of four stimuli in Figure 1a. One stimulus was presented at a time, and the task was to decide whether its (say) left-hand element curved to the left or to the right. We found that speed and accuracy of performance was markedly impaired when the irrelevant (right-hand) element 
A.

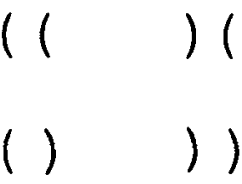

)(

)

c.<smiles>[Hg]</smiles>

B.

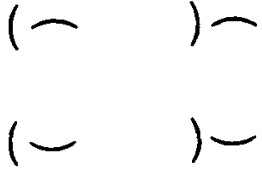

requiring distribution of attention across elements rather than focusing of attention required in selective attention tasks. The goal here was to achieve a convergence on the concept of perceptual organization through a number of independent experimental procedures.

D.

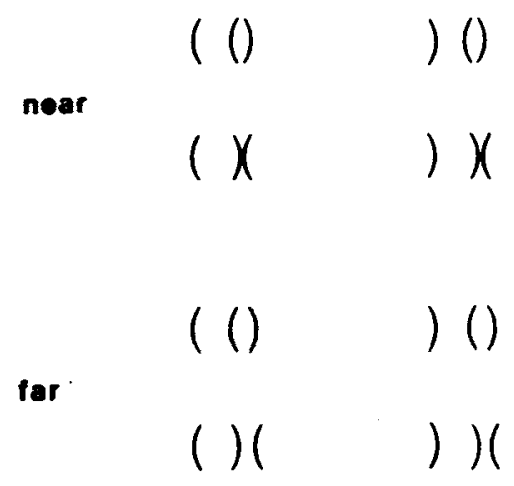

Figure 1. (A, B) The stimulus sets ased by Pomerantz and Garner (1973). (C) The nearest and widest interelement spcaings used in Experiment 1. (D) The two stimulus sets used in Experiment II.

varied unpredictably in direction of curvature, compared to when it was the same for all stimuli in the presentation sequence. Since the only difference between these two tasks was in the irrelevant element (i.e., whether it varied or remained constant), it was clear that this element was being attended to despite its irrelevance to the tasks. Assuming that attention would not have been paid voluntarily to irrelevant information, it follows that the distribution of attention across both elements was involuntary or mandatory. When this experiment was repeated with the stimuli in Figure $1 \mathbf{b}$, no such impairment was found. We could therefore conclude that (1) organizational processes could be measured by success in selective attention tasks; that (2) by this criterion the elements of the stimuli in Figure la grouped while those in Figure $1 \mathrm{~b}$ did not; and that (3) the grouping process is of a mandatory nature.

\section{Purpose of Experiment}

One purpose of the research reported below was to use this measure of grouping to explore the effects of some stimulus factors which traditionally have been assumed to affect perceptual organization. In particular, we were interested in the effects of

\section{EXPERIMENT I \\ EXPERIMIENTI}

(a) interelement spacing (or proximity), and (b) the addition of extra stimulus elements which might lead to a perceptual reorganization of a stimulus array. A second purpose was to explore whether organizational effects that appear with selective attention tasks

This first experiment had several purposes, the first of which was to examine the role of element proximity in the grouping process. The results of the Pomerantz and Garner experiment suggested that proximity was a necessary, but not sufficient, condition for grouping. We argued that proximity was not a sufficient condition since the elements in Figure $1 \mathrm{~b}$ did not group even though those in Figure 1a, which had the same spatial separation, did group. We argued that proximity was a necessary condition because as the two elements comprising each stimulus are moved further apart. perceptual grouping at some point would surely break down. Experiment I below demonstrates that this is correct and that for most subjects the breakdown is complete by the time the elenients in Figure 1a are more than $\mathbf{4} \mathrm{deg}$ apart in visual angle.

The fact that grouping breaks down over large distances, while reassuring, is not of great interest since it could hardly have turned out otherwise: certainly, if the elements were spaced so widely that they could not both be seen within a single fixation, no grouping could occur. A more interesting question, however, is not whether grouping disappears but how it disappears as spacing increases. Answering this question formed the second purpose of Experiment I. Recall that the measure of grouping being used is the difference in performance between tasks where the irrelevant element reniains constant for all stimuli in the presentation sequence and tasks where it varies from stimulus to stimulus, independently of (orthogonally to) the variation in the relevant element which the subject is required to discriminate. Let us call tasks of the lirst type "constant context" tasks and those of the second type "orthogonal context" tasks. As this nomenclature suggests, the irrelevant element can be thought of as providing a context within which the relevant element must be discriminated. When performance in the orthogonal context task is worse than that on the constant context task, the elements are said to group. As elements that group when closely spaced are moved further apart, pertormance in the orthogonal context task should 
iniprove; this would indicate that variation in the irrelevant element becomes less distracting the less it groups with the relevant one. On the other hand, performance on the constant context task should remain stable as the elements are separated, since there should be no distraction or interference from the irrelevant, unchanging element at any proximity. The findings from Experiment $I$ do not conform exactly with this seemingly straightforward set of predictions, and the way in which these results deviate suggests a moditication of the way in which we have conceptualized the grouping process.

A third purpose of this experiment was to compare performance on selective attention tasks with performance on condensation tasks requiring distributed attention. If grouping implies a failure of selective attention, then tasks which do not require sclective attention should not be adversely affected by grouping. Typically, tasks which require that attention be paid to two sources are more difficult than those where only one must be processed, but this might not happen if the two sources are configural elements which are grouped together and processed as a single unit regardless of task requirements. On the other hand, as two elements become less able to be grouped (e.g., as they are moved apart), distributed attention should become more difficult.

A fourth and relatively minor purpose of Experiment I was to examine order-of-exposure effects in perceptual grouping. Because of the particular task used here and in the Pomerantz and Garner experiment (speeded card sorting), the two elements comprising each stimulus do not become visible to subjects at the same instant. It night be expected that irrelevant information might be harder to ignore if it preceded the relevant information, since it night be possible in the latter case for processing to be partially or fully completed before irrelevant information became available. Experiment I exanined this question explicitly.

\section{Method}

This experiment contained three major experimental factors: (a) the different discrimination tasks used; (b) the proximities of the elements comprising the stimuli for these tasks; and (c) the order of exposure of the elements within each stimulus.

\section{Discrimination tasks}

The four tasks in this experiment required discrimination among two-element stimuli like those shown in Figure 1a; these tasks differed as to which particular stimuli were used in each task and what response was to be made to each stimulus in the task. The actual procedure used was speeded card sorting.

Constant context tasks (2). In these tasks, the subject was required to discriminate between two stimuli that differed only with respect to the right-hand elements. Just two such tasks are possible, and both were used: ( ) vs. ( ( and ) ) vs. ) (. To illustrate, consider the first of these tasks. The subject was handed a deck of cards, each of which contained a two-element stimulus. The deck contained 32 cards, with 16 showing the stimulus () and 16 the stimulus ( 1 . The deck was shuftled, and the task was to sort the deck out into two piles, one for each stimulus, as quickly as possible. For this particular discrimination, the left-hand element is irrelevant, since it is the same for the stimuli ( ) and ( (. Thus only the right-hand element must be processed for the task to be performed. This task, along with its complement ) ) vs. ) (, is called a constant context task because the irrelevant element, which creates a conligural context for the relevant one, was constant for each of the 32 stimuli in the deck.

Orthogonal context task (1). This task, like the two above, required subjects to discriminate between stimuli on the basis of their right-hand element. Now, however, the irrelevant, left-hand element varied from stimulus to stimulus in the deck, independently of (orthogonally to) the variation in the relevant element. Thus, the subject was handed a shuffled deck of 32 cards containing eight copies of each of the four stimuli in Figure 1a. The stimuli () and ) ) were to be placed into one pile and the stimuli ( ( and ) (into the other pile. Again, the irrelevant left-hand element could be safiely ignored.

Condensation task (1). This task, unlike the constant and orthogonal context tasks, was not a selective attention task, since it required that attention be distributed across both the left-and right-hand elements. This task involved the same deck of stimuli as the orthogonal context task, only now the stimuli () and ) (were to be sorted into one pile and the stimuli (( and $)$ ) into the other. Here the subject was required to respond to the joint state of the two elements. He had to distribute his attention to integrate or condense information from two sources in order to perform the task, such as by determining whether the two elements were parallel, or whether they formed a vertically symmetrical pattern.

\section{Proximities}

The stimuli used were of the type shown in Figure 1a, oniy with a variety of interelement spacings. Six proximities were sampled; the narrowest and widest are shown to scale for the stimulus () in Figure lc. The stimuli were typed on $6.3 \times 8.9 \mathrm{~cm}$ white cards using the left and right parenthesis keys of an electric pica typewriter. The interelement spacings corresponded to $.5,1,2,4,8$, and 16 spaces on the typewriter. Each parenthesis was approximately $3 \mathrm{~mm}$ tall, and each space on the typewriter corresponded to about $2.6 \mathrm{~mm}$. At a typical viewing distance of $30 \mathrm{~cm}$, these stimuli subtended visual angles ranging from .25 to $8.0 \mathrm{deg}$. To prevent a contounding of proximity with absolute location of the relevant element on the card in the selective attention tasks, the right-hand element was typed in the same place on all cards at all proximities; thus, increases in spacing were achieved by moving the left-hand element toward the left edge of the card.

\section{Order of Exposure}

Some subjects preterred to sort cards holding the deck in their left hands and others in their right hands. These preferences were fairly independent of reported handedness. When the deck is held in the left hand, cards are pulled off the top of the deck from left to right, thus exposing the left side of the next card in the deck before the right side. In the constant context and orthogonal context tasks. where the relevant element is on the right-hand side, this would mean that the irrelevant element would become visible before the relevant one for these subjects, and vice versa for subjects holding the deck in the right hand. Since this was potentially an important source of variation, two groups of subjects were used, differing in preferred hand.

\section{Design and Procedure}

Each subject performed 96 sortings ( 4 tasks $\times 6$ proximities $x 4$ replications). These 90 sortings were arranged into four blocks, each of which constituted one complete replication. The first block was considered practice and was not used in the data analysis. Within each block, there was further blocking by proximity so that all four tasks at any one proximity were performed in immediate succession, followed by all four at the next proximity, and so on. The order of the four lasks was counterbalanced within subjects. 
A.

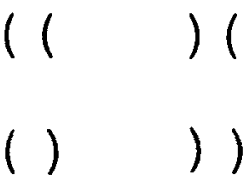

C.

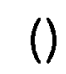

8.

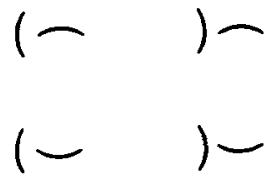

(a) interelement spacing (or proximity), and (b) the addition of extra stimulus elenents which might lead to a perceptual reorganization of a stimulus array. A second purpose $w$ as to explore whether organizational effects that appear with selective attention tasks would also manifest thenselves in other types of tasks requiring distribution of attention across elements rather than focusing of attention required in selective attention tasks. The goal here was to achieve a convergence on the concept of perceptual organization through a number of independent experimental procedures.

\section{EXPERIMENT I}

D.

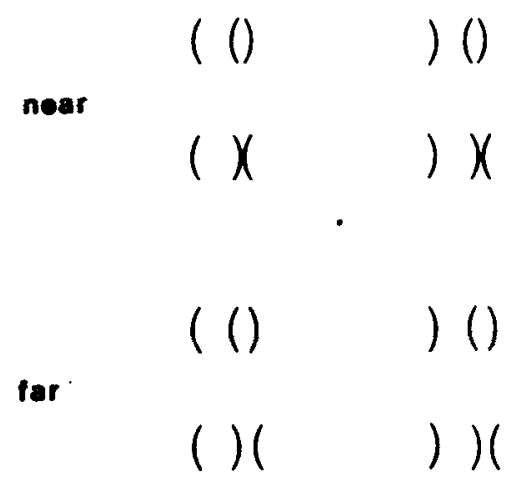

Figure 1. (A, B) The stimulus sets used by Pomerantz and Garner (1973). (C) The neareat and wheat interelement spcaings nsed in Experiment I. (D) The two etimulus sets used in Experiment II.

varied unpredictably in direction of curvature, compared to when it was the same for all stimuli in the presentation sequence. Since the only difference between these two tasks was in the irrelevant element (i.e., whether it varied or remained constant), it was clear that this element was being attended to despite its irrelevance to the tasks. Assuming that attention would not have been paid voluntarily to irrelevant information, it follows that the distribution of attention across both elements was involuntary or mandatory. When this experiment was repeated with the stimuli in Figure $1 b$, no such impairment was found. We could therelore conclude that (1) organizational processes could be measured by success in selective attention tasks; that (2) by this criterion the elements of the stimuli in Figure 1 a grouped white those in Figure $1 \mathrm{~b}$ did not; and that (3) the grouping process is of a mandatory nature.

\section{Purpose of Experiment}

One purpose of the research reported below was to use this measure of grouping to explore the effects of some stimulus factors which traditionally have been assumed to affect perceptual organization. In particular, we were interested in the effects of

This lirst experiment had several purposes, the first of which was to examine the role of element proximity in the grouping process. The results of the Pomerantz and Garner experiment suggested that proximity was a necessary, but not sufficient, condition for grouping. We argued that proximity was not a sufficient condition since the elements in Figure $1 \mathrm{~b}$ did not group even though those in Figure 1a, which had the same spatial separation, did group. We argued that proximity was a necessary condition because as the two elements comprising each stimulus are moved further apart. perceptual grouping at sone point would surely break down. Experiment I below demonstrates that this is correct and that for most subjects the breakdown is complete by the time the elements in Figure la are more than 4 deg apart in visual angle.

The fact that grouping breaks down over large distances, while reassuring, is not of great interest since it could hardly have turned out otherwise: certainly, if the elements were spaced so widely that they could not both be seen within a single fixation, no grouping could occur. A more interesting question, however, is not whether grouping disappears but how it disappears as spacing increases. Answering this question formed the second purpose of Experiment I. Recall that the measure of grouping being used is the difference in performance between tasks where the irrelevant element renains constant for all stimuli in the presentation sequence and tasks where it varies from stinulus to stimulus, independently of (orthogonally to) the variation in the relevant element which the subject is required to discriminate. Let us call tasks of the tirst type "constant context" tasks and those of the second type "orthogonal context" tasks. As this nomenclature suggests, the irrelevant element can be thought of as providing a context within which the relevant element must be discrininated. When performance in the orthogonal context task is worse than that on the constant context task. the elements are said to group. As elements that group when closely spaced are moved further apart, performance in the orthogonal context task should 
iniprove; this would indicate that variation in the irrelevant element becomes less distracting the less it groups with the relevant one. On the other hand, performance on the constant context task should remain stable as the elements are separated, since there should be no distraction or interference from the irrelevant, unchanging element at any proximity. The findings from Experiment $I$ do not conform exactly with this seemingly straightforward set of predictions, and the way in which these results deviate suggests a modilication of the way in which we have conceptualized the grouping process.

A third purpose of this experiment was to compare performance on selective attention tasks with performance on condensation tasks requiring distributed attention. If grouping implies a failure of selective attention, then tasks which do not require sclective attention should not be adversely affected by grouping. Typically, tasks which require that attention be paid to two sources are more difficult than those where only one must be processed, but this night not happen if the two sources are configural elements which are grouped together and processed as a single unit regardless of task requirements. On the other hand, as two elements become less able to be grouped (e.g., as they are moved apart), distributed attention should become more difficult.

A fourth and relatively minor purpose of Experiment $I$ was to examine order-of-exposure effects in perceptual grouping. Because of the particular task used here and in the Pomerantz and Garner experiment (speeded card sorting), the two elements comprising each stimulus do not become visible to subjects at the same instant. It might be expected that irrelevant information might be harder to ignore if it preceded the relevant information, since it night be possible in the latter case for processing to be partially or fully completed before irrelevant information became available. Experiment I examined this question explicitly.

\section{Method}

This experiment contained three major experimental factors: (a) the different discrimination tasks used; (b) the proximities of the elements comprising the stimuli for these tasks; and (c) the order of exposure of the elements within each stiniulus.

\section{Discrimination tasks}

The four tasks in this experiment required discrimination among two-element stimuli like those shown in Figure 1a; these tasks differed as to which particular stimuli were used in each task and what response was to be made to each stimulus in the task. The actual procedure used was speeded card sorting.

Constant context tasks (2). In these tasks, the subject was required to discriminate between two stimuli that differed only with respect to the right-hand elements. Just two such tasks are possible, and both were used: ( ) vs. ( ( and ) ) vs. ) (. To illustrate, consider the tirst of these tasks. The subject was handed a deck of cards, each of which contained a two-elentent stimulus. The deck contained 32 cards, with 16 showing the stimulus () and 16 the stimulus ( (. The deck was shuffled, and the task was to sort the deck out into two piles, one for each stimulus, as quickly as possible. For this particular discrimination, the left-hand element is irrelevant, since it is the same for the stimuli () and ( (. Thus only the right-hand element must be processed for the task to be performed. This task, along with its complement) ) vs. ) (, is called a constant context task because the irrelevant element, which creates a configural context for the relevant one, was constant for each of the 32 stimuli in the deck.

Orthogonal context task (1). This task, like the two above, required subjects to discriminate between stimuli on the basis of their right-hand element. Now, however, the irrelevant, left-hand element varied from stimulus to stimulus in the deck, independently of (orthogonally to) the variation in the relevant element. Thus, the subject was handed a shuffled deck of 32 cards containing eight copies of each of the four stimuli in Figure 1a. The stimuli ( ) and ) were to be placed into one pile and the stimuli ( ( and ) ( into the other pile. Again, the irrelevant left-hand element could be safely ignored.

Condensation task (1). This task, unlike the constant and orthogonal context tasks, was not a selective attention task, since it required that attention be distributed across both the left- and right-hand elenents. This task involved the same deck of stimuli as the orthogonal context task. only now the stimuli ( ) and ) (were to be sorted into one pile and the stimuli $(($ and $))$ into the other. Here the subject was required to respond to the joint state of the two elements. He had to distribute his attention to integrate or condense information from two sources in order to perform the task, such as by determining whether the two elements were parallel, or whether they formed a vertically symmetrical pattern.

\section{Proximities}

The stimuli used were of the type shown in Figure 1a, only with a variety of interelement spacings. Six proximities were sampled; the narrowest and widest are shown to scale for the stimulus () in Figure $l c$. The stimuli were typed on $6.3 \times 8.9 \mathrm{~cm}$ white cards using the left and right parenthesis keys of an electric pica typewriter. The interelement spacings corresponded to $.5,1,2,4,8$, and 16 spaces on the typewriter. Each parenthesis was approximately $3 \mathrm{~mm}$ tall. and each space on the typewriter corresponded to about $2.6 \mathrm{~mm}$. At a typical viewing distance of $30 \mathrm{~cm}$, these stimuli subtended visual angles ranging from .25 to $8.0 \mathrm{deg}$. To prevent a contound ing of proximity with absolute location of the relevant element on the card in the selective attention tasks, the right-hand element was typed in the same place on all cards at all proximities; thus, increases in spacing were achieved by moving the left-hand element toward the left edge of the card.

\section{Order of Exposure}

Some subjects preferred to sort cards holding the deck in their left hands and others in their right hands. These preferences were fairly independent of reported handedness. When the deck is held in the left hand. cards are pulled off the top of the deck from left to right, thus exposing the left side of the next card in the deck before the right side. In the constant context and orthogonal context tasks. where the relevant element is on the right-hand side, this would mean that the irrelevant element would become visible before the relevant one for these subjects, and vice versa for subjects holding the deck in the right hand. Since this was potentially an important source of variation, two groups of subjects were used, differing in preferred hand.

\section{Design and Procedure}

Each subject performed 96 sortings $(4$ tasks $\times 6$ proximities $\times 4$ replications). These 96 sortings were arranged into four blocks, each of which constituted one complete replication. The first block was considered practice and was not used in the data analysis. Within each block. there was further blocking by proximity so that all four tasks at any one proximity were performed in immediate succession, followed by all four at the next proximity, and so on. The order of the four tasks was counterbalanced within subjects. 
The order of the six proximities was counterbalanced across subjects. with each subject within a group receiving the proximities in a different order.

For each sorting, the subject was handed a well-shuffled deck of cards, face up, along with an instruction card indicating which stimuli were contained in the deck and which stimuli were assigned to which response pile. Each subject was allowed to decide the left-right placement of the two piles for himself. When the subject was ready, the experimenter initiated the trial by pressing a footswitch which started a timer and served as an audible cue for the subject to begin sorting. The timer was stopped by the experimenter at the moment the subject placed the last card in the deck. The subject was instructed to sort as fast as possible while keeping errors to a minimum.

\section{Subjects}

A total of 12 subjects were used, including 6 who preferred sorting from the left hand and 6 from the right. The subjects averaged 21 years of age and included six males. Each was paid for serving in a single $2 \cdot \boldsymbol{h}$ session.

\section{Results}

Sorting time results are shown in Figure 2. Data are shown separately for the two groups of subjects which differed in preferred hand. The data have been pooled across the two constant context tasks since pertormance did not differ significantly between them and since the two tasks are theoretically equivalent.

Analysis of variance on sorting times showed that tasks $[F(3,30)=21.2]$, proximities $[F(5,50)=22.8]$, and most importantly the Tasks by Proximities interaction $[F(15,150)=25.7]$ all varied significantly beyond the .001 level. Order of exposure, a
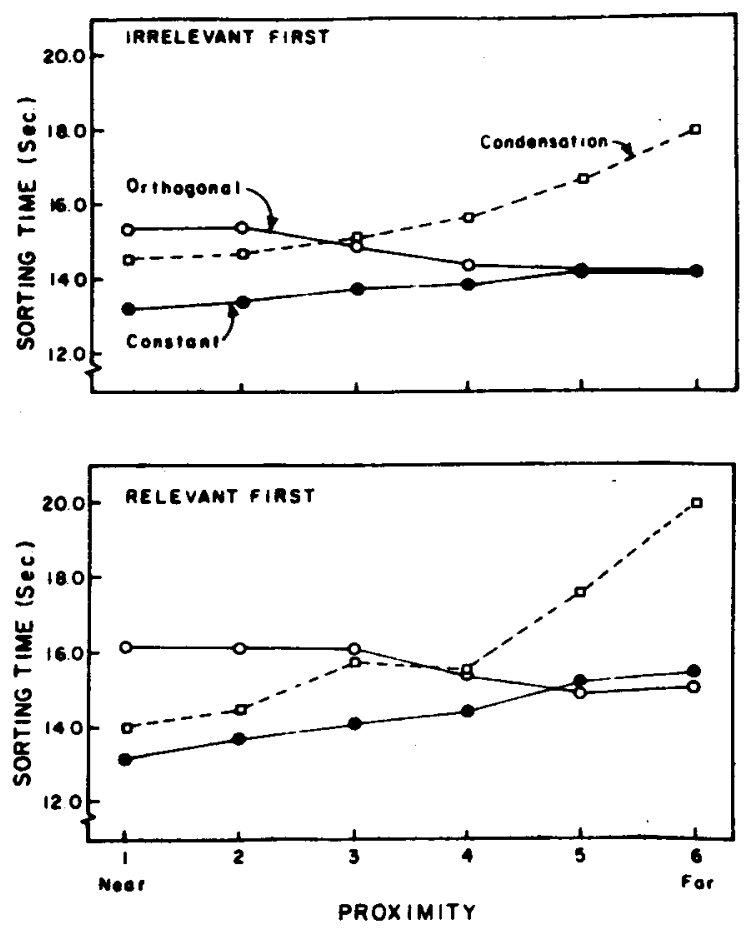

Figure 2. Mean corthes then per dock for the three procening tauks as a function of interelement proximily. between-subjects factor, did not approach significance $[F(1,10)<1]$, nor did any interactions involving this factor. Thus, it did not matter whether the relevant or irrelevant element became visible first in the selective attention tasks, and so a potential methodological problem with the card-sorting technique can be dismissed.

The form of the Tasks by Proximities interaction in Figure 2 is clear: the constant context and condensation tasks were performed more slowly with increased spacing between the elements, while the orthogonal context tasks were performed faster. Separate analyses on these three tasks showed significant effects of proximity for each $[F(5,55)=$ $12.88,11.16,38.08$ for the constant context, orthogonal context. and condensation tasks, respectively; all $\mathrm{p}<.001$ ], with the linear component accounting for $74 \%, 71 \%$, and $97 \%$ of the variance, respectively.

The degree to which performance on the orthogonal context task was slower than that on the constant context task constitutes our measure of grouping. This measure was strongly positive $(2.6 \mathrm{sec})$ at the closest spacing, where 11 of 12 subjects showed a positive value. As spacing increased, this measure declined monotonically toward and slightly beyond zero $(-0.2 \mathrm{sec})$ at the widest spacing, where 6 of 12 subjects showed a positive value. The condensation task began at the closest spacing, showing an intermediate level of difficulty, being slower than the constant context but faster than the orthogonal context tasks for 11 of 12 subjects. Performance deteriorated rapidly with increased spacing until, at the widest spacing, this task constituted the single most difficult condition in the entire experiment for all 12 subjects.

As is typical in card-sorting experiments of this type, too few errors were made to allow a formal analysis. The few errors that were made tended to form a pattern similar to that reported above, with the slower tasks showing more errors.

\section{Proximity}

\section{Discussion}

At the closest element separations used, strong grouping effects were found: performance was much slower in the orthogonal context than in the constant context tasks. As the elements were spaced more widely, grouping diminished monotonically until it had disappeared entirely at a 4-deg separation. Thus this experiment has shown that it is possible to demonstrate proximity effects with performance measures of perceptual grouping rather than with subjective report or judgment.

The absolute visual angle at which grouping disappeared entirely cannot be stressed for two reasons. First, precise visual angles are neither controlled nor measured in a card-sorting experiment. 
The distance of the subject's eyes from the stimuli can and does vary with this technique. Notice, however, that any attempt by the subject to compensate for changes in visual angle by altering his viewing distance would have served to attenuate the observed effects of elemint proximity, not to exaggerate them. Thus the presunce of proximity effects indicates either that subjects did not attempt such compensation, or that they could not do so effectively. Second, it is possible that it is not absolute separation but relative separation of elements which determines their grouping. That is, grouping may persist out to very wide visual angles if the elenients are expanded proportionally in size.

\section{Nature of the Grouping Process}

The discovery of a proximity effect in grouping was not of great interest, per se. What was interesting, however, was the precise nature of this effect. It was found that as the irrelevant, distracting element was moved away from the relevant one in the orthogonal context task, performance improved. This was not surprising since the interfering effect of irrelevant information could only be expected to diminish at greater distances. However, in the constant context tasks, moving the irrelevant element away from the relevant one actually made performance suffer.

How is this finding to be explained? If the irrelevant element in the constant context condition had simply not been processed, then moving it away from the relevant one should have produced no effect. If this element had been attended to at close proximities, or had interfered by its mere presence with processing of the relevant one via lateral masking (Shaw, 1969; Townsend, Taylor, \& Brown, 1971), then moving the irrelevant element away should have facilitated perfornance. What kind of processing would have led, however, to impaired performance?

Grouping as an automatic process. Two possible explanations of this outcome can be offered. Under the first, grouping is conceptualized as an automatic involuntary operation, perhaps handled by a primitive, preattentive process in the perceptual system (Neisser, 1967) which is not under strategic control. This preattentive process is influenced by certain physical parameters of the stimulus such as proximity, similarity, etc. Its effect is to integrate physically separate stimulus elements into a single perceptual code. The consequence of this integration is that elements become "glued" together and cannot be responded to independently or attended to selectively. At close proximities, then, the subject must deal with these two-element stimuli as whole patterns. Thus, in the orthogonal context task he must cope with four alternative stimuli, while in the constant context tasks he must deal with only two. The number of alternative stimuli has a well-known effect in reaction time experiments, slowing performance as the number increases, even when the number of alternative responses is held constant (Posner, 1964). As the elements are moved apart, they become "unglued" and the subject is more able to deal with them as separate entities. Thus, the orthogonal context task changes in effect from a four-alternative to a two-alternative task as separation increases, and this explains the improvement in performance that was observed for that task. In the constant context task, however, there are only two effective alternative stimuli regardless of whether the subject treats the elements as wholes or as separate entities. When the elements were far apart, the subject might have attempted to process stimuli wholistically even though preattentive processes did not require it. We can assume that attending to both elements should have become more difficult as they were separated (perhaps since both elements could not be fixated foveally), and this assumption is justitied by the deterioration in performance in the condensation task, which will be discussed below. Thus, performance in the constant context task actually got worse as the elements were separated.

Grouping as an active strategy. An alternative explanation would hold that the perceptual grouping effects are due not to an involuntary, preattentive process but rather to a deliberate strategy of integrating elements into wholes. The hypothesis here is that attention is paid to irrelevant, redundant information because doing so somehow facilitates discrimination. This notion has a distinctly counterintuitive flavor, but similar ideas have been advanced in studies on the perception of verbal materials to account for the so-called "word superiority effect" and the "word-letter phenomenon" (Johnston \& McClelland, 1973, 1974; Reicher, 1969; Wheeler, 1970). According to this hypothesis, the stimuli () and ( (are somehow more discriminable from each other than the stimuli) and (, even though there is no additional information which makes the stimuli more different in a formal sense. In any case, the subjects deliberately attended to both elements in the constant context task because it aided discrimination, but doing so became more difficult as the elements were separated by greater distances, since it became harder to encode both elements in a single fixation. This explains why performance got worse in the constant context tasks as proximity decreased. Why, under this explanation, did performance improve with separation in the orthogonal context task? Since grouping is a voluntary process, the subject would decide, even at the closest proximities, not to group the elements but to process them separately in order to avoid having to deal with four alternative patterns rather than just two alternative elements. However, the mere presence of an irrelevant element might have impaired discrimination of the relevant one, perhaps through 
lateral masking or perhaps through response competition. This would then explain why pertormance improved in the orthogonal context tasks.

The data from this experiment cannot differentiate between these two potential explanations, although they could be differentiated by other experimental manipulations. For example, the automatic process explanation of grouping relies on the larger number of alternative stimuli to account for poor performance in the orthogonal context tasks. If this is correct, then increasing the number of alternatives in this task beyond four (by using stimuli composed of more than just two elements) should slow performance even further. Second, performance in the constant context task should be compared with a no-context task (i.e., discrimination of single elements). If grouping of an element with its context improves its discriminability, as argued under the strategic process explanation, then removing context should hurt performance. Pilot data have been collected on both of these tests, and the results have supported the notion that grouping, at least with the kinds of elements shown in Figure 1, is a strategic process.

\section{Performance in Condensation Tasks}

Not unexpectedly, performance on the condensation task grew worse at wider element separations. Since the nature of this task required that attention be paid to both elements, either of two approaches could have been employed: processing both elements wholistically as a single unit or processing the two elements separately and perhaps sequentially. We assume that at the closest spacings the first alternative would have been used, either because the subject had to process wholistically (if grouping is a mandatory operation) or chose to (if grouping is an optional strategy). If this assumption is correct, there are two plausible explanations for the proximity effect on this task: reduced efficiency of grouping at greater element separations or a shift away from grouping toward the presumably less efficient strategy of processing elements separately.

As mentioned earlier, condensation tasks are generally more difficult than selective attention or filtering tasks (Posner, 1964). That is, it is usually easier to process one source and ignore a second source than to attend to both. However, if both sources must be attended to anyway (because they are grouped) in both tasks, then it might be expected that condensation tasks would actually be no harder than selective attention tasks. The results presented above support this reasoning. In fact, at the two closest proximities, the condensation task was actually faster than the orthogonal context task for 11 of the 12 subjects in the experiment. Why this reversal should have occurred is not entirely clear. Perhaps it was because the condensation task would allow the subject to have abstracted a global feature of the stimuli (such as symmetry of elements) rather than a more local one (direction of curvature of the right-hand side).

\section{EXPERIMENT II}

While Experiment I stressed the importance of proximity in grouping, it could be argued that proximity per se is not a primary factor in perceptual organization. The Gestalt psychologists emphasized that whether two elements, A and B, would group perceptually would be determined not just by the relationship of $A$ and $B$ alone, but also by whatever else was present in the perceptual field. That is, the organization of a percept is structured to take into account the entirety of perceptual field and is thus not responsive just to local factors. Translating this reasoning into experimental terms, it should be possible to break down the grouping between $A$ and $B$ by adding a third element, $C$. For example, $C$ could beconie grouped with $B$, leaving $A$ perceptually isolated. We attempted to demonstrate just such an effect with the stimuli in Figure 1d.

Looking at any one of the stimuli in Figure 1d, the size and spacing of the two left-hand elements was the same as that at the third closest spacing $(1 \mathrm{deg})$ in Experiment I, where powerful grouping effects were observed. The question was whether these effects would remain after a third element was added which appeared to cause a perceptual "reorganization" of the elements. As shown in the figure, two different spacings $(.25$ and $.50 \mathrm{deg})$ between this third element and the second element were used.

The method and procedure of this experiment were the same as in Experiment I, except that the left-hand rather than the right-hand element was relevant (which, as shown in Pomerantz and Garner, 1973, should not matter). Thus, the task was to sort the stimuli on the basis of the left-hand element while the two right-hand elements, which provided an irrelevant context, either remained constant for all stimuli or varied orthogonally to the relevant element in the presentation sequence. No condensation task was employed. A fresh group of 10 subjects was used.

The results are shown in Table 1 . The important feature to notice is the difference in sorting time

Table 1

Sorting Time Results for Two Stimulus Sets in Experiment $\mathrm{I}^{*}$

\begin{tabular}{|c|c|c|c|c|c|}
\hline & & & \multicolumn{3}{|c|}{ Task } \\
\hline & & : & $\begin{array}{l}\text { Constant } \\
\text { Context }\end{array}$ & $\begin{array}{l}\text { Ortho- } \\
\text { gonal } \\
\text { Context }\end{array}$ & $\begin{array}{c}\text { Group- } \\
\text { ing } \\
\text { Effect }\end{array}$ \\
\hline $\begin{array}{l}\text { Spacing of } \\
\text { Third }\end{array}$ & Near & $(.25 \mathrm{deg})$ & 13.19 & 13.34 & .15 \\
\hline Element & Far & $(.50 \mathrm{deg})$ & 13.46 & 13.56 & .10 \\
\hline
\end{tabular}

-Sorting times are means in seconds per deck of 32 cards. The grouping effect is measured by the arithmetic difference between the orthogonal context and constant context sorting times. 
between the orthogonal context and constant context tasks, which is our measure of grouping. When the third element was closely spaced to the original pair of elements, this difference averaged $.15 \mathrm{sec} /$ deck; at the wider spacing, it averaged $.10 \mathrm{sec}$. Neither of these differences was signiticant. The corresponding difference in Experiment 1 was $1.54 \mathrm{sec} /$ deck (more than 10 times larger), and no subject in that experiment (or in the Pomerantz and Garner experiment) showed a grouping effect as small as this $.10-.15$-sec difference. Thus, grouping that exists between two elements can be reduced or perhaps eliminated entirely by adding a third element to the perceptual field, even though this third element does not physically intervene between the first two.

To summarize, it is clear that proximity has a potent effect in perceptual grouping, even when assessed by performance measures. Absolute proximity per se does not appear to be of overriding importance in perceptual grouping, since other factors in the stimulus field can determine whether grouping will occur at a given proximity. What remains unclear is the exact mechanism by which grouping operates. On the basis of earlier work (Pomerantz \& Garner, 1973), it appeared that grouping was the result of an automatic process, under preattentive control, which governed the formation of perceptual units. If two elements were grouped, this meant that they had been integrated into a single unit and that the individual elements so grouped could not be attended to selectively or processed independently. The results presented above have suggested another interpretation: Grouping may be the result of an optional strategy, which is chosen in certain tasks because, for some reason, grouping of elements can improve their discriminability. The "laws" of perceptual organization would thus become rules which allow, rather than dictate, the construction of perceptual units.

\section{REFERENCES}

Egeth, H. Selective attention. Psychological Bulletin, 1967, 67, 41-57.

GARNER, W. R. The processing of information and structure. Potomac, Md: Lawrence Erlbaum Associates, 1974.

Garner, W. R., \& Felfoldy, G. L. Integrality of stimulus dimensions in various types of information processing. Cognitive Psychology, 1970, 1, 225-241.

Johnston, J. C., \& MCClelland, J. L. Visual factors in word perception. Perception \& Psychophysics, 1973, 14, 365-370.

Johnston, J. C., \& McClelland, J. L. Perception of letters in words: Seek not and ye shall find. Science, 1974, 184, 1192-1194.

NeIsser, U. Cognitive psychology. New York: AppletonCentury-Crafts, 1967.

Pomerantz, J. R., \& Garner, W. R. Stimulus configuration in selective attention teshs. Penception \& Psychophysics, 1973, 14, 565-569.

Posner, M. 1. Information reduction in the analysis of sequential tasks. Psychological Review, 1964, 71, 491-504.

Reicher; G. M. Perceptual recognition as a function of meaningfulness of stimulus materials. Joumal of Experimental Psychology, 1969, 81, 275-280.

Shaw, P. Processing of tachistoscopic displays with controlled order of characters and spaces. Perception \& Psychophysics, $1969,6,257-266$.

Townsend, J. T., TAylor, S. G., \& Brown, D. R. Lateral masking for letters with unlimited viewing time. Perception \& Psychophysics, 1971, 10, 375.378.

WERTHEIMER, M. Untersuchungen zur Lehre von der Gestalt. Psychologische Forschung, 1923, 4, 301-350. (In W. D. Ellis (Ed.), A source book of Gestalt psychology. New York: Humanities Press, 1967.)

Wheeler, D. D. Processes in word recognition. Cognitive Psychology, 1970, 1, 59-85.

(Received for publication June 30, 1975; accepted August 6, 1975.) 